\title{
27
}

\section{Methods for Performance Monitoring and Traffic Measurements at Cell Level in ATM networks}

\author{
F. Lozano and M. J. Manso
}

ALCATEL Standard Eléctrica. Research and Development Center. Ramírez de Prado, 5. 28045 MADRID, Spain. email : flr@ seiv10.rpi.ses.alcatel.es

\begin{abstract}
ATM Network Management requires the system to calculate and to control all the traffic and performance parameters at cell level. Since the old methods and algorithms used to monitor at call level are not applicable at cell level due to the much shorter time scale, and the more stringent performance requirements, new algorithms and methods have to be developed to estimate and to control these parameters. This paper describes solutions to this problem, providing accurate methods to monitor in short time each one of the parameters used to define the cell transfer performance and traffic in every part of an ATM network, switching node and end-points of the different paths: Cell Transfer Rate and Cell Loss Ratio. These methods allow to do ATM Performance Management controlling any QOS degradation at cell level.
\end{abstract}

Keyword Codes: C.4; C.2.3; C.2.1

Keywords: Performance of Systems; Network Operation; Network Architecture and Design

\section{INTRODUCTION}

The introduction of Broadband Integrated Services Digital Networks (B-ISDN) based on Asynchronous Transfer Mode (ATM) [1,2], as well as the new services to be provided, make Network Management [3,4] a key issue in communication networks, as they become faster, more complex, and more flexible with greater requirements for high network availability.

With the introduction of ATM networks, new performance and traffic parameters (Cell Loss Ratio, Cell Insertion Ratio, etc.) have been defined at cell level, for the management, operation and maintenance of such networks. These parameters must be monitored on-line, both end-to-end and in the switching node.

Performance monitoring at cell level bumps into new troubles compared with 
management at connection level and call level, as these latter are closer to whatever has been done elsewhere. In this manner, parameters to be controlled at the cell level present the problem of being of a low incidence (because of the high level of requirements for performance parameters, sometimes in the order of magnitude of $10^{-10}$ ), which leads to a large observation period before finding a significant occurrence value. However, a quick reaction is necessary in order to be able to control the possible degradation (the high cell rate obliges the system to detect anomalies and to react in a very short time). Therefore, due to these two characteristics, traditional methodology and algorithms are worthless here, and new ones are necessary to monitor and calculate performance monitoring parameters at cell level.

For the performance management at cell level on ATM paths, traffic flow over an ATM path is defined by means of the Successfully Transferred Cells parameter, and the following five performance parameters related to the latter are usually established: Cell Loss Ratio, Cell Missinsertion Rate, Cell Error Ratio, Cell Transfer Delay, and Cell Delay Variation.

This paper is focused on the first two parameters. Procedures for the calculation and the monitoring of these parameters at different points of the network, end-to-end, as well as in every path, and inside a switching node are proposed. In the second section of this paper, the first parameter (Cell Transfer Rate) is studied, proposing an appropriate value for the duration of the time interval needed for measuring this parameter. In the third section, the second parameter (Cell Loss Ratio) is studied. The last section presents the most important conclusions achieved with this study.

\section{CELL TRANSFER RATE}

Actual traffic flow is defined by means of the Cell Transfer Rate parameter. Cell Transfer Rate is defined as the ratio of the number of successful cell transfer outcome events during a specified time to this time interval duration. This parameter should be calculated and monitored over Virtual Paths, Virtual Channels, ATM Paths, and internal links inside the Switching node.

The Usage Parameter Control (UPC) takes care of the load monitoring in VPs and VCs, i.e., it assures that the load is kept within a certain range of values, without calculating an exact value for the load. Many papers can be found on this matter [5]. Nevertheless, if a value for the cell transfer rate has to be obtained (i.e., not a monitoring of the parameter, but a calculation of its exact value), OAM cells might be the solution by adding up, for the cell transfer rate computation, the cells arriving between two consecutive OAM cells.

For the case of ATM paths (linking as they usually do Switching Nodes, and internal links inside a Switching Node), a procedure for calculating the traffic load 
value is counting the number of busy cells (volume count) at incoming or outgoing end-points during a certain period of time. The complexity of this procedure resides in the choice of the value of this period of time. This time period depends on two considerations as they are reaction time, and reliability:

- On the one hand, this measurement time must accomplish to be short enough to allow preventive/reactive control actions when necessary.

- On the other hand, however, this time has to be long enough to obtain a significant estimate of the actual traffic load.

Consequently, the measurement time should fulfill the two above conditions. The selection of the measurement time is an important point, that is going to be developed in the next subsection for two cases: an ATM link, and internal links inside the switching node.

For the case of external ATM links, F3 flow OAM cells could be used to calculate the value of the Cell Transfer Rate, by adding the value of the Total User Cell Number [4] of 90 consecutive OAM cells inserted every 1024 cell time units.

\subsection{Choosing a statistically significant measurement time}

The case of an ATM Link is going to be considered first, and secondly the case of internal links inside the switching node.

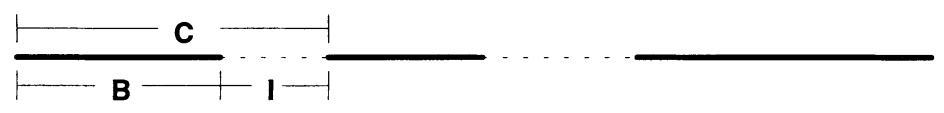
B: Busy period
I : Idle period
C : Cycle time

Figure 1. M/D/1 server cycle time.

\section{1.a. External ATM link (ATM Path)}

Consider a 155 Mbps ATM link, external to a Switching Node. Traffic flow in such a link comes from the composition of other flows generated at the independent sources that share that link. At cell level, this flow can be modeled as the outgoing flow from an $M / D / 1$ system, which is known to represent an upper bound for this flow [6]. No burst level is considered because of the limited buffering of the ATM switch (taking into account the small capacity of buffering of this kind of switch, no long bursts are likely to be produced, because cells are lost instead of buffered). In this model, the service time is the time for transmitting a cell at the capacity of the link. 
An unbiased estimate of the load can be obtained from samples of the volume count taken at regeneration points. Recall that in a M/D/1 model, the beginnings of busy periods are regeneration points. So, a cycle time of the process alternates a busy and an idle period, starting with a busy one (see figure 1 above).

The average length of the time cycle, $\mathbf{C}$, is given [7] by

$$
C=\frac{1}{\lambda(1-\rho)}
$$

where $\lambda$ is the cell arrival rate and $\rho$ is the server load in erlangs.

Figure 2 below plots cycle time (measured in cell units) vs. server load for a $\mathrm{M} / \mathrm{D} / 1$ system representing a $155 \mathrm{Mbps}$ link. The service time equal to one cell time unit (the time needed for the transmission of a cell) implies $\lambda=\rho$ (i.e., the figure has to be interpreted in this way: for each value of $\rho$, the value of the cycle time of the system is on the corresponding curve with the same value for $\lambda$ and $\rho$ ). Looking at this figure, it can be seen that, at nominal load (0.8), the average $\mathbf{C}$ is under ten cell units.

\section{Cycle time}

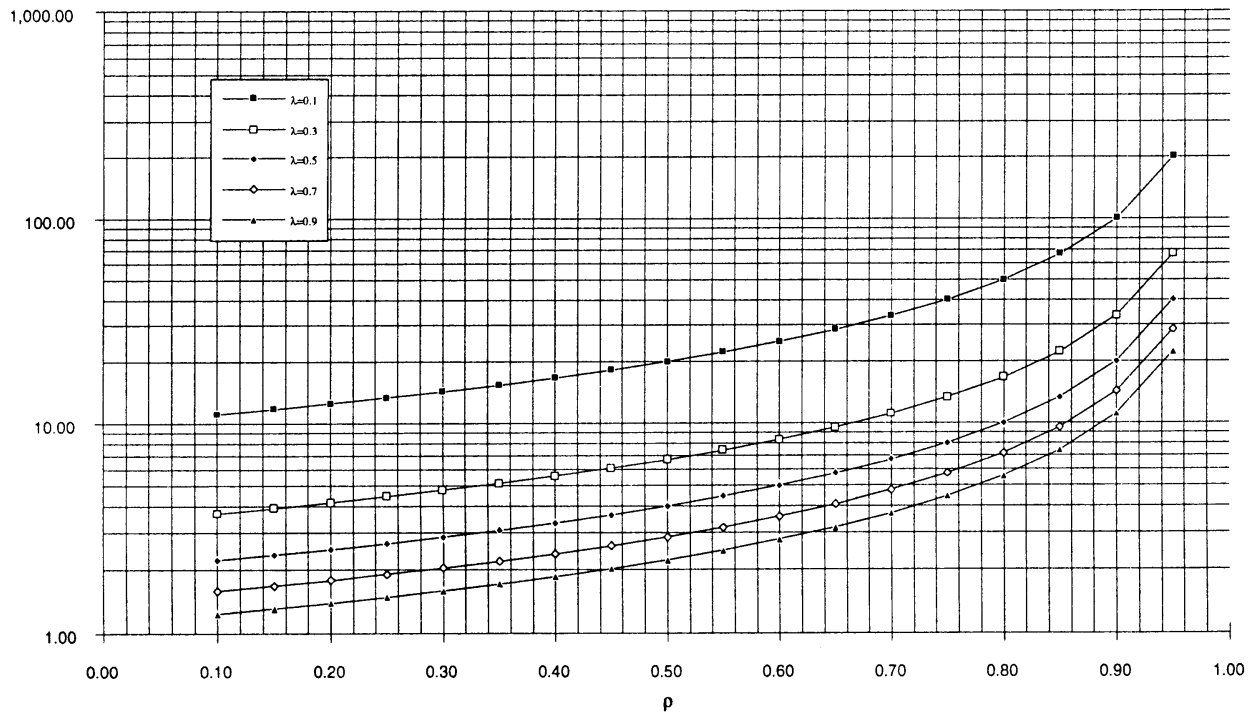

Figure 2: Variation of the $M / D / 1$ average cycle time.

Two aspects have to be taken into account when looking for a reliable measurement time: 
a) Trying to make negligible the fact that neither the beginning nor the ending of a busy period are known, it has been thought a good approach to take a multiple of the average cycle time as measurement period. So, let N.C be the measurement elementary period, where $\mathrm{N}$ represents a number to be later on determined, and $\mathrm{C}$ the average cycle time. A sample of the value of the load is taken in this elementary period.

b) The other point to deal with is the accuracy: the number of samples to be taken. To assure a significant value for the load estimate, a certain number of samples (M) of the value of the parameter to be estimated should be taken for each period of measurement. Load is then estimated as the average of those samples. Therefore, the measurement time has M elementary periods.

Taking $\mathrm{M} \cdot \mathrm{N} \cdot \mathrm{C}$ as the measurement time period would lead to a greater period than the one we need. Because the two above points have to be accomplished at the same time, it is quite enough to take the minimum measurement time as the product of $\mathrm{C}$ and the maximum value between $\mathrm{M}$ and $\mathrm{N}$. In general, $\mathrm{M}$ is a higher value than $\mathrm{N}$ (that could be close to 30 ), so we are going to take as a minimum measurement time M.C.

Since $1 /(1-\rho)$ is the mean number of busy cells per cycle time, and $\rho^{2}$ is the variance of the number of busy cells per cycle time, the relative error, $E$, will be

$$
\frac{Z_{\alpha / 2}}{M^{1 / 2}} \leq E
$$

Therefore, the number of samples to be taken must satisfy

$$
M>\left(\frac{Z_{\alpha / 2}}{E}\right)^{2}
$$

It can be then shown that a confidence interval of about $99.99 \%$ (and an error of 0.1 ) can be obtained with a number of samples equal to 1475 .

Taking into account that for a $155 \mathrm{Mbps}$ link at nominal load the average $\mathrm{C}$ is under ten cell time units, as said before, these values would take a measurement time of approaching 14750 cell time units, around 42 milliseconds.

\section{1.b. Internal Switching Node links}

The ATM Switching Node that is going to be considered in this case is ALCATEL's [8]. The switching element of such a node is made up of 16 inlets and 16 outlets. Traffic flow in an outlet can be modeled [9] as a Batch-Geo/D/1, with 16 inlets and a service time equivalent to one cell time unit, for which the above 
expression of the average cycle time $\{1\}$ also applies.

In this switch, outlets are grouped, forming what is called a Quad Link, (made up of four links at $155 \mathrm{Mbps}$ ). Following this, the Quad link is modeled as a Batch-Geo/D/4 system. Therefore, the average cycle time (sum of a busy period plus an idle period) is limited by:

$$
C=\frac{1}{\lambda(1-\lambda)}
$$

where $\lambda$ is the batch arrival rate:

$$
\lambda=1-q^{16}
$$

and

$$
q=1-\frac{4 \cdot \rho}{16}
$$

being $\rho$ the load on a single link (expressed in erlangs).

Taking 0.7 as the maximum value for $\rho$, that leads to a cycle time of 22.8 time cell units. Applying the same rationale for obtaining the value of $M$ as in the previous section, it is then easy to calculate that a confidence interval of about $99.99 \%$ (and an error of 0.1 ) can be obtained with a number of samples equal to 1475. These values would take a measurement time of $95 \mathrm{msec}$.

\subsection{Considerations on the response time}

The buffer in the multiplexer has been considered as the place in the switching node where a delay in taking actions would be the most critical [10] (because a huge amount of cells are lost in case the capacity of this buffer is surpassed).

Several simulation runs have been carried out to estimate the sensitivity of this buffer to sudden load increases in the external links in front of it.

In each run, the transient period has been calculated with a common buffer size of 150 and changing the load from 0.8 to 0.9 erlangs in all its external ATM links during a period of $100 \mathrm{msec}$.

Out of those simulation runs can be seen how an expected buffer overflow increases from $10 \mathrm{E}(-12)$ (dimensioning value) to $10 \mathrm{E}(-5)$ in a very short time (few hundreds of milliseconds) if no actions are taken to download the links. From these values it can be deduced that cell losses are expected in $300 \mathrm{msec}$, and not before. Therefore, a maximum reaction time of about $250 \mathrm{msec}$. would permit the 
Cell Lost Ratio to be kept under the expected $10 \mathrm{E}(-12)$ value (because the reaction has to be produced before an important number of cells are lost).

\subsection{Summary}

The time to calculate the measurement has to range between the values calculated in previous sections. As has been explained already, the time needed to perform the measurement has to be long enough to allow the detection of significant occurrence values; the calculations performed in subsections 2.1.a and 2.1.b have allowed the minimum values for this time to be found. On the other hand, the time to perform the measurement has to be small enough as to allow the reaction in time of corrector mechanisms; in subsection 2.2 the rationale for the obtainment of a maximum value has been presented.

Therefore, the measurement time has to be at least the values obtained in sections 2.1.a and 2.1.b (42 and $95 \mathrm{msec}$, respectively) and, at the same time, to be lower than $250 \mathrm{msec}$. (calculated as a good maximum value of response time in section 2.2). From all the above considerations, it has been concluded that 250 msec. is a good value for the measurement time for short time load calculation in Switching Node ATM links (external or internal Quad Links).

\section{CELL LOSS RATIO (CLR)}

The Cell Loss Ratio parameter is defined by [11] as the ratio of lost cells to the total number of transmitted cells in a population of interest. Also in [11] a method to calculate this parameter end-to-end for VPs and VCs is proposed, using OAM cells. Each OAM cell contains information of the number of transmitted cells containing user information $(\mathrm{Nt})$ since the last OAM cell. Counting the number of received cells $(\mathrm{Nr})$, the $\mathrm{CLR}$ will be given by

$$
\frac{(N t-N r)}{N t}
$$

This method seems to work well, though it makes neither reference on how to monitor the CLR (not to have an excessive CLR), nor on what should be the time between two consecutive OAM cells.

Since this method is only applicable to the calculation of end-to-end CLR for VCs and VPs, a new method is necessary to calculate and to monitor the CLR in a switch of the Switching node, to know at which exact switch an excessive CLR occurs.

Given the high level of requirements for this parameter, the problem is that it would take too much time to obtain its exact value, being impossible to correct 
the possible faults in time to avoid a severe degradation of the QOS. That means that it is impossible to measure this parameter in real time. Then, the key issue becomes to monitor CLR, i.e., to watch over it in order to detect if its value is under a specified threshold. Following is a proposal of a method to monitor the CLR in each switch. This method does not take into account different Cell Loss priorities, but it could be also applicable to that case.

In an ATM Switching node, cell losses mostly happen due to output buffer overflow at nodes when many cells show up almost simultaneously. Several studies on this matter show that they occur in bursts [15]; that is, when a cell is lost, the probability that a new cell loss occurs in the next time period is very high. That is what has been called in this model a Burst of losses or Loss Burst. In order to characterize such a burst of cell losses, several papers propose using the Interrupted Poisson Process (IPP). This arrival process is alternately turned On for an exponentially distributed time and then turned Off for another exponentially distributed time [12]. In our case, one exponential distribution will be used to model the inter-losses time inside the burst (what is going to be referred to as I.L.T.) and the other one to characterize the time inter bursts of losses. In the figure 3 a scheme of this process is represented, with the parameters defining it (where the small boxes inside loss bursts represent a single loss event).

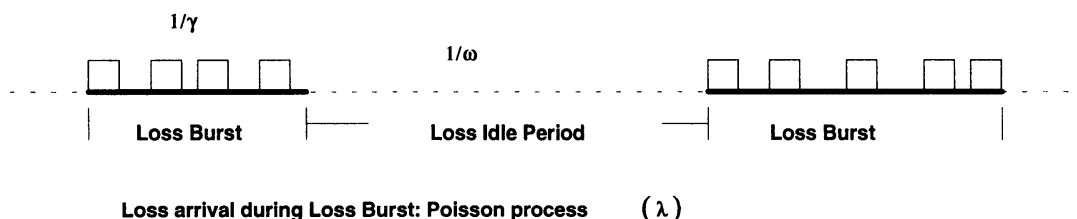

Figure 3. Loss arrival process (IPP).

where:

$1 / \gamma=$ Mean Loss Burst duration

$1 / \omega=$ Mean Loss Idle Period duration

$\lambda=$ Mean Cell Loss in a time unit during the Loss Burst

The inter-loss time distribution will be given by:

$A(t)=$ probability $(I . L \cdot T . \leq t)=k_{1}\left(1-\exp \left(-r_{1} t\right)\right)+k_{2}\left(1-\exp \left(-r_{2} t\right)\right)$

with:

$$
r_{1}=\frac{1}{2}\left\{\lambda+\omega+\gamma+\sqrt{(\lambda+\omega+\gamma)^{2}-4 \omega \lambda}\right\}
$$




$$
\begin{aligned}
& r_{2}=\frac{1}{2}\left\{\lambda+\omega+\gamma-\sqrt{(\lambda+\omega+\gamma)^{2}-4 \omega \lambda}\right\} \\
& k_{1}=\frac{\lambda-r_{2}}{r_{1}-r_{2}} \quad k_{2}=1-k_{1}
\end{aligned}
$$

In this system, the value of the CLR during a complete cycle C (a loss period and an idle period) as a function of the parameters defining the model is:

$$
C L R=\frac{\frac{\lambda}{\gamma}}{\left(\frac{1}{\gamma}+\frac{1}{\omega}\right) \cdot \rho}
$$

The duration of a regeneration period in an IPP is the time between the beginning of two consecutive Loss Bursts, that is $C=1 / \gamma+1 / \omega$.

\begin{tabular}{ccccc} 
\# Incoming links & $\begin{array}{c}\text { \# Output links groups } \\
\text { (\# links per group) }\end{array}$ & $\rho$ & $\lambda$ & $1 / \gamma$ \\
\hline 8 & $4(1)$ & 6.4 & 1.4 & 2.4 \\
8 & $1(4)$ & 6.4 & 1.3 & 2.3 \\
16 & $8(2)$ & 12.8 & 5.4 & .8 \\
16 & $4(4)$ & 12.8 & 5.2 & .8 \\
\hline
\end{tabular}

Table 1. Values for $\lambda$ and $\gamma$.

To give an idea of the time necessary for measuring this parameter, the value of $\mathrm{C}$ has to be known. To estimate the value of $\mathrm{C}$, several simulations with different combinations of output link groupings, each one at 0.8 Erlangs, and different number of inlets with exponential arrival and constant service, have been carried out using a method to get rare events: Restart method [13]. The results (see table 1 ) show the different values of $1 / \gamma$ and $\lambda$, $\rho$ being the total load in the switch. Knowing this values, $\omega$ can be calculated from $\{3\}$ to obtain the corresponding CLR:

$$
\omega=\frac{\gamma}{\frac{\lambda}{C L R^{*} \rho}-1}
$$


For the values of table $1, \omega$ is in the order of magnitude of $10^{-12}$ (about $2 \mathrm{E}-12$ and 2,9E-12 for the cases of 8 and 16 incoming links, respectively). So, C is in the orders of $10^{11}$, which leads, considering links at $155 \mathrm{Mbps}$, to a necessary observation time of several years. This time is clearly too big to be useful. A possible alternative could be in using sliding windows with a very large size and a short time of jumping time. Even though assuming a jumping time of 1 day and a window size of 1 year (one day could be a good reaction time), the number of active windows in each instant would be 365 . This value is impossible to manage due to the great amount of memory and processor effort it would require.

Two methods to monitor the CLR and, at the same time to have a reasonable reaction time, are proposed below using windows of 15 minutes size. The choice of this time is to have an appropriate time for reaction and to assure at least a loss burst within a window, with the above values of $\omega$ and $\gamma$. But, these methods can be used with other sizes of windows, with only changing the equivalence between windows and time. Windows with a loss burst (loss windows) and windows without cell losses (idle windows) are considered in any case.

It has to be recalled that the aim of these methods is to monitor the CLR (to detect an excessive CLR) and not to give an exact value for this parameter.

\subsection{Method of checking only the number of Lost Cells depending on the number of Idle Windows (LC/IW method)}

The first method would consist in, once the number of idle windows $\left(\mathrm{N}_{\mathrm{iw}}\right)$ between consecutive loss windows is known, determining the maximum allowed number of lost cells in a loss window in order to maintain the required CLR (taking into account the number of idle windows already passed). I.e., the parameter that is considered as independent variable is the time elapsed between two loss windows, and the variable that depends on it and that is going to be controlled is the number of lost cells in the loss window. The process is as follows.

The first step is counting the number of idle windows between the last window of losses and the following one. After, in order to determine the number of lost cells that can be allowed in every loss window to keep the value of the CLR under the maximum allowed figure, the parameter $\lambda$ of the IPP has to be obtained. For that, the parameters $\omega$ and $\gamma$ are given the following values:

- $1 / \omega$ is taken equal to $\left(\mathrm{N}_{\mathrm{iw}}+1\right) * 15$ minutes (what is not more than express, in minutes, the idle period that has been measured in idle windows),

- and $1 / \gamma=3$, which is the largest value that appeared in the simulations (i.e., the maximum time that any switch needs in order to go back to a normal 
situation after an overflow occurs).

The value of $\lambda$ can be then obtained from $\{4\}$ using these values and the value required for the CLR which we want to check out:

$$
\lambda=C L R \cdot \rho \cdot\left(\frac{\gamma}{\omega}+1\right)
$$

With the obtained $\lambda$ value, and knowing that the number of cells in a loss burst follows a Poisson distribution of mean $\lambda / \gamma$, then it is possible to calculate the number of cells $\mathrm{X}$ thus as

$$
\mathrm{P}[\text { Number of lost cells }(\lambda / \gamma)>\mathrm{X}] \leq 0.01
$$

As it is difficult to assert in 15 minutes whether the CLR is in accord with the expected one, and as it may not even have too much sense (because the necessary data may not be the value for the CLR, but knowing if its value is kept under a threshold), the chosen statistic measures whether the CLR is excessive with a certain probability (following the above expression, a probability of $99 \%$ ) for the case of the number of lost cells exceeds a certain limit, X. Figure 4 shows the $X$ values for $\rho=6.4$ erlangs (dashed line) and 12.8 erlangs (solid line).

\section{X vs Niw}

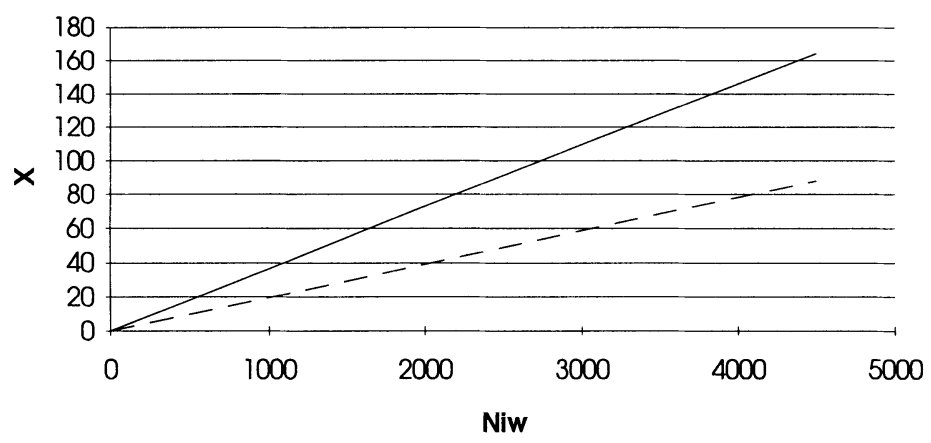

Figure 4: $\mathrm{X}$ calculated in function of $\mathrm{N}_{\mathrm{iw}}$.

Summarizing, the procedure following which this method works is the 
following:

1. The number of idle windows between two consecutive loss windows is counted (i.e., the time between consecutive loss windows, $1 / \omega$, is obtained).

2. The value of the duration of the loss burst, $1 / \gamma$, is predetermined (it should be chosen as the maximum time that the switch needs in order to go back to a normal situation after an overflow occurs).

3. The average value of the Cell Loss Ratio produced in a time unit during the Loss Burst $(\lambda)$ is calculated from all these values ( $\omega$ and $\gamma$ ), using the expression $\{5\}$.

4. The number of lost cells allowed in the burst of losses (X) to assure with a specified probability that the CLR is not higher than the specified one is calculated.

In this way, whenever a loss burst occurs in a window, the number of lost cells is counted; as soon as the number of lost cells exceeds the corresponding value of $\mathrm{X}$ calculated with $\mathrm{N}_{\mathrm{iw}}$, an alarm notification of Excessive Cell Loss Ratio has to be reported.

\subsection{Method of checking the number of Lost Cells and the number of Idle Windows (LC\&IW Method)}

The second method would consist in putting maximum limits to the values of $\lambda$ and $\omega$ (let say $\lambda_{0}$ and $\omega_{0}$, respectively) and then controlling both the number of lost cells in the loss window and the number of idle windows in the following way:

- to calculate the maximum number of lost cells that can be allowed in a loss window in order to ensure that the real $\lambda$ does not exceed $\lambda_{0}$

- to calculate the minimum number of idle windows $\left(\mathrm{N}_{\mathrm{iw}}\right)$ that are needed between two consecutive windows of losses in order to ensure that the real $\omega$ does not exceed $\omega_{0}$, (what is the same than to say that the rate of arrival of windows of losses has to be lower than the prefixed one: $\omega_{0}$ ).

To put it another way, what is being done is to establish a maximum threshold for the average number of lost cells in a unit of time in the loss burst $(\lambda)$, and a minimum one for the average time between bursts of losses $(1 / \omega)$, and to look for the values under which the total number of lost cells in the window and the rate of loss windows have to be in order to accomplish these thresholds.

The limit values, $\lambda_{0}$ and $\omega_{0}$, can be obtained by means of simulations for 
obtaining the required CLR. (For instance, in Table 2 are shown the values of $\lambda_{0}$ and $\omega_{0}$ for a CLR of $10^{-12}$.) Of course, these thresholds are as much stringent as the value of CLR becomes lower.

The process for calculate the maximum number of lost cells in a burst of losses and the minimum number of idle windows is as follows.

If the inter losses time inside a loss burst is supposed to be exponential, the number of lost cells during the loss burst, $x$, follows a Poisson distribution of mean $\lambda / \gamma$. If $\lambda$ is supposed to vary continuously, we have a bidimensional distribution:

$$
f(\lambda, x)=\frac{e^{-\lambda / \gamma} \cdot(\lambda / \gamma)^{x}}{x !}
$$

To calculate the maximum number of cells in a loss burst, $\mathrm{X}$, maintaining at the same time the value of the $\lambda$ parameter under the limit value $\lambda_{0}$, we operate in the following way:

$$
\begin{gathered}
P\left\{\lambda \leq \lambda_{0} / x=X\right\}=1-P\left\{\lambda>\lambda_{0} / x=X\right\}= \\
1-\frac{\int_{\lambda_{0}}^{\infty} f(\lambda, X) d \lambda}{f_{x}(X)}=1-\frac{\int_{\lambda_{0}}^{\infty} \frac{e^{-\lambda / \gamma} \cdot(\lambda / \gamma)^{X}}{X !} d \lambda}{\int_{0}^{\infty} \frac{e^{-\lambda / \gamma} \cdot(\lambda / \gamma)^{X}}{X !} d \lambda}
\end{gathered}
$$

and, integrating by parts:

$$
P\left\{\lambda \leq \lambda_{0} / x=X\right\}=1-\sum_{I=0}^{X} \frac{e^{-\lambda_{0} / \gamma} \cdot\left(\lambda_{0} / \gamma\right)^{I}}{I !}=P\left\{x\left(\lambda_{0}\right)>X\right\}
$$

being $x\left(\begin{array}{ll}\lambda & 0\end{array}\right)$ the number of lost cells, with mean $\lambda_{0} / \gamma$.

We operate in an analogous way to calculate the minimum number of idle windows, Y. Knowing that the time of the idle period follows an exponential distribution of mean $1 / \omega$, and $\omega$ is supposed to vary continuously, we have the bidimensional distribution:

$$
g(\omega, t)=\omega \cdot e^{-\omega t}
$$


And, therefore, expressing the time in terms of the number of idle windows $\left(\mathrm{N}_{\mathrm{iw}}\right)$, as well as $\omega$ :

$$
\begin{aligned}
& P\left\{\omega \leq \omega_{0} / N_{i w}=Y\right\}= \\
& =1-\frac{\int_{0}^{\infty} \omega \cdot e^{-\omega \cdot Y} d \omega}{\int_{0}^{\infty} \omega \cdot e^{-\omega \cdot Y} d \omega}=1-e^{-\omega_{0} \cdot Y}=P\left\{N_{i w}\left(\omega_{0}\right) \leq Y\right\}
\end{aligned}
$$

where $N_{i w}\left(\begin{array}{ll}\omega & 0\end{array}\right)$ represents the number of idle windows, with mean $1 / \omega_{0}$.

With these above distributions it is possible to calculate $\mathrm{X}$ and $\mathrm{Y}$ values for obtaining a certain probability of $\lambda$ and $\omega$ not exceeding their respective thresholds, $\lambda_{0}$ and $\omega_{0}$.

The next table (Table 2) shows the required values for $X$ and $Y$ to get, with an accuracy of $99 \%$, that $\lambda$ and $\omega$ values do not exceed $\lambda_{0}$ and $\omega_{0} .\left(1 / \omega_{0}\right.$ and $Y$ are expressed in number of 15 minute windows).

\begin{tabular}{ccccc} 
\# Incoming links & $1 / \omega_{0}$ & $\lambda_{0}$ & $\mathrm{X}$ & $\mathrm{Y}$ \\
\hline 8 & 1340 & 1.5 & 14 & 10 \\
16 & 2194 & 5.5 & 22 & 9 \\
\hline
\end{tabular}

Table 2. Values for the number of lost cells (X) and the number of idle windows (Y).

Summarizing, the steps following which the method works are the following:

1. The values for $\lambda$ and $\omega$ parameters are predetermined for the target value of $\operatorname{CLR}\left(\lambda_{0}\right.$ and $\omega_{0}$, respectively).

2. Once a maximum value for the average cell loss during a unit of time in the loss burst is fixed, $\lambda_{0}$, the maximum number of cells allowed in the loss burst $(\mathrm{X})$ is calculated to assure with a specified probability that the $\lambda$ parameter does not exceed the threshold $\lambda_{0}$.

3. Once a minimum value for the mean time between two consecutive burst of losses is fixed, $\omega_{0}$, the minimum number of idle windows allowed $(\mathrm{Y})$ is 
calculated to assure with a specified probability that the $\omega$ parameter does not exceed the threshold $\omega_{0}$ (i.e., the mean time between loss bursts is higher than the established minimum).

Then, when a loss window appears, an Excessive CLR notification will be generated if $\mathrm{N}_{\mathrm{iw}}$ is less than $\mathrm{Y}$, or if the number of lost cells exceeds $\mathrm{X}$ in each switch.

The value of the CLR can be estimated every day (for instance), by means of the values of $\mathrm{N}_{\mathrm{iw}}$ and the number of lost cells in the loss windows.

These methods could be applied to control CLR end-to-end in an ATM path using a counter at the end-point. For VPs and VCs, CLR can be calculated using OAM cells, as the ratio of the sum of lost cells $(\Sigma(\mathrm{Nt}-\mathrm{Nr}))$ between two consecutive OAM cells (which are inserted during the 15 minutes) to the sum of transmitted cells $(\Sigma \mathrm{Nt})$ between the same OAM cells. The number of OAM cells inserted during 15 minutes depends on the total load over the VP or VC. For a VP at $50 \mathrm{Mbps}$, the number of OAM cells would be 107,400 inserting an OAM cell every 1024 user cells.

Requirements on this parameter are not referred to single connections, but to the average of a set of them. Therefore the calculation of the values should be done over all active connections within each 15 minute period.

\section{CONCLUSIONS}

Since the methods and algorithms used before to monitor at call level are not applicable in ATM network management at cell level, due to the high performance requirements and the high cell rate, new methods to calculate and to monitor the different cell transfer performance and traffic parameters are needed.

Some of these methods have already been proposed, but without specifying times or taking into account estimation accuracy. This paper, using these methods and new methods here described, specifies the times which allow the degradation of some cell transfer performance parameters to be detected in short time, and simultaneously to get an accurate estimation of the performance measurements in a larger time.

A methodology to calculate an appropriate time to monitor the traffic (Cell Transfer Rate parameter) over external ATM links as well as internal links of an ATM Switching node has been specified.

Also two methods to detect an excessive Cell Lost Ratio and, at the same time, to have a reasonable reaction time have been proposed: the LC/IW Method and the LC\&IW Method. 
These methods allow to perform QOS Observation and Performance Management at ATM level according to the CCITT [15] using a Current Data associated with every kind of element of the network with an observation time of 15 minutes. This makes possible to detect the exact portion of the network at which a QOS degradation has occurred.

\section{REFERENCES}

[1] "Asynchronous Transfer Mode. Solution for Broadband ISDN." Martin de Prycker. Ellis Horwood. 1991.

[2] CCITT Recommendation I.362 "B-ISDN ATM Adaptation Layer (AAL) Functional Description".

[3] CCITT Recommendation M.3200 "TMN Management Services: Overview".

[4] CCITT Recommendation I.610 "OAM principles of B-ISDN access".

[5] "Queuing, Performance and Control in ATM". J. W. Cohen , C. D. Pack, Editors. 13th International Teletraffic Congress, Copenhagen 1991. North Holland Studies in Telecommunication, Vol. 15.

[6] Performance Evaluation and design of multiservice networks. COST 224. Management Committee of the COST 224 project. Editor J.W. Roberts. CEE.

[7] "Queueing Systems. Vol. I. Theory". L. Kleinrock. John Wiley \&Sons. 1975.

[8] "Switching Network Architecture for ATM based Broadband Communications". M.A.Henrion, K.J. Schrodi, D. Boettle, M. De Somer, D. Dieudonné. ISS'90, Stockholm, May 1990.

[9] "Queuing Models Applying to a class of ATM Multiplexers". A.Guerrero \& F. Lozano. 13th International Teletraffic Congress, Copenhagen 1991. North Holland Studies in Telecommunication, Vol. 14.

[10] "Performance Evaluation Methods Applicable to an ATM Multi-Path SelfRouting Switching Network". G.H. Petit, A. Buchheister, A.Guerrero, P Parmentier. 13th International Teletraffic Congress, Copenhagen 1991. North Holland Studies in Telecommunication, Vol. 14.

[11] CCITT Recommendation I.356, "B-ISDN ATM Layer cell transfer performance".

[12] "The Interrupted Poisson Process as an Overflow Process". A. Kuczura. Bell System Technical Journal. Vol. 52. No. 3. March 1973.

[13] "RESTART: A method for Accelerating Rare Events Simulations". M. Villen-Altamirano, J. Villen-Altamirano. 13th International Teletraffic Congress, Copenhagen 1991. North Holland Studies in Telecommunication, Vol. 15.

[14] CCITT Recommendation Q.822 "Description for Q3 Interface: Performance Management".

[15] "Performance Design Method for ATM Networks and Systems". T.Yokoi, Y.Yamamoto, Y.Fujii, T.Betchaku. NTT Review. Vol. 4. July 1992. 